Draft of the paper:

Anna Esposito, Antonietta M. Esposito, Italia Cirillo, Lina Panfilo, Filomena Scibelli, Mauro Maldonato, Carl Vogel

(2017) "Differences between hearing and deaf subjects in decoding foreign emotional faces". In Proceedings of the

8th IEEE International Conference on Cognitive InfoCommunication, Debrecen, Hungary, 11-14 September 2017,

\title{
Differences between hearing and deaf subjects in decoding foreign emotional faces
}

\author{
Anna Esposito ${ }^{1}$, Antonietta M. Esposito ${ }^{2}$, Italia \\ Cirillo $^{1}$, Lina Panfilo ${ }^{1}$, Filomena Scibelli ${ }^{1}$ \\ ${ }^{1}$ Department of Psychology, Università della Campania \\ "Luigi Vanvitelli", and IIASS, Caserta, Italy \\ ${ }^{2}$ Istituto Nazionale di Geofisica e Vulcanologia, Sez. di \\ Napoli Osservatorio Vesuviano, Italy \\ iiass.annaesp@tin.it, antonietta.esposito@ingv.it
}

\author{
Mauro Maldonato ${ }^{3}$, Carl Vogel ${ }^{4}$ \\ ${ }^{3}$ Dipartimento di Scienze Umane, Università della \\ Basilicata, Italy \\ ${ }^{4}$ School of Computer Science and Statistics, Trinity College \\ Dublin, Ireland \\ maldonato@gmail.com,vogel@tcd.ie
}

\begin{abstract}
This work investigates the ability of deaf subjects to correctly label foreign emotional faces of happiness, sadness, surprise, anger, fear, and disgust, in comparison with typically hearing ones. The experiment involved 14 deaf (signing) and 14 hearing subjects matched by age and gender. The emotional faces were selected from the Radboud Database. The results show significant difference between the two groups, with deaf performing significantly poorly in the decoding accuracy and intensity ratings of disgust, surprise, and anger. Considerations are made on the effects of the social and cultural context to leverage the universality of emotional facial expressions.
\end{abstract}

Keywords - deaf and hearing subjects; facial emotional stimuli; emotion decoding accuracy; mean intensity ratings

\section{INTRODUCTION}

There are not too many investigations comparing the ability of deaf and typically hearing subjects to decode emotional facial expressions belonging to different cultures. These comparisons have been neglected, due to the largely accepted belief that facial expressions of emotions share universal features among humans no matter the social, organizational, and cultural context in which they are expressed. This belief has been debated by recent studies showing that there are social, cultural, and circumstantial factors playing a role in supporting the human ability to decode emotional faces. In this context it has been shown that cultural, communication, and mood disorders such as depression, as well as alcoholism, and personality traits play an important role in decoding emotional labels [14], [15], [16], [17], [20], [28], [31].

When deafness comes to play, emotional expressions are an integral part of the communication mode and are largely considered in the deaf culture. In particular, it is well known that signing people exploit facial expressions as meaningful communicative signs in combination with other meaningful body and hands movements [5], [7], [32], [33], [34]. Given the large range of meanings and usages of facial expressions in sign languages, it is natural to investigate how emotional faces are discriminated from communicative signs and whether and to what extent some facial emotional expressions, such as those associated to the six primary emotions [11], [12], have universal emotional meanings without reference to individual social contexts or social culture. The studies comparing hearing and deaf subjects' ability to decode the facial expressions of the six primary emotions report inconsistent findings that show either no substantial differences among the two groups [23], or poorer performance of the deaf in comparison to their peers [9], [22]. Generally, these studies considered deaf and hearing children and stimuli of facial emotional expressions belonging to the same cultural group. What if the facial stimuli belong to a different, even though close, culture?

The present study aims to test the above hypothesis comparing the ability of Italian deaf and hearing subjects in decoding Dutch facial emotional expressions selected from the Radboud Database [27]. The adult database contains the facial emotional expressions of 39 Caucasian Dutch adults (19 female), expressing neutral emotional feelings, as well as anger, sadness, fear, disgust, surprise, happiness, and contempt. The actor portraits assume three different gaze directions, frontal, left, and right, and five camera angles (see [27] for details).

\section{MATERIALS AND PROCEDURES}

\section{A. Participants}

28 Italian subjects were recruited for this experiment of which fourteen were signing deaf ( 8 females) and fourteen typically hearing subjects (6 females). The deaf subjects were 
recruited at ENS (Ente Nazionale Sordi) of Caserta (Italy), with the permission of the president of the association. Deaf were aged from 35 to 55 years (mean age $=48.36$ years, $S D= \pm 4.21$ ) and typically hearing subjects' age was matched (mean age $=47.50$ years, $\mathrm{SD}= \pm 3.21$ ) with them.

\section{B. Materials}

24 photographs of posed facial expressions taken from Radboud Database were selected [27]. Only frontal stimuli (frontal gaze direction and frontal camera angle) were considered expressing the 6 basic emotions of happiness, sadness, surprise, disgust, fear and anger [11], [12]. The selected stimuli are listed in Table I. Each emotion was expressed by two female and male faces. Fig. 1 shows an example of the female and male stimuli expressing sadness and happiness respectively.

\section{Procedures}

A suitable laboratory setting was created, free of distractions and disturbing events. Each participant was briefed on the required task and if volunteered to do it, asked to complete and sign the consent form. Subsequently, she was put in front to a computer screen and asked to watch and assign one of the six abovementioned emotional categories or the "no emotion" or "another emotion" label, to each of the 24 selected emotional faces randomly played through the Superlab® software by appropriately selecting the associated keyboard's key. Then participants were asked to rate the intensity degree of the expressed emotion on a Likert scale from 0 (absence of any emotional intensity) to 9 (very intense emotion). Each subject was tested in a private room and assisted by two experimenters.

TABLE I

\begin{tabular}{|c|c|}
\hline \multicolumn{2}{|c|}{$\begin{array}{l}\text { List of emotional facial expressions selected for the reported } \\
\text { experiment from the Radboud Database [27] }\end{array}$} \\
\hline disgust & $\begin{array}{l}\text { Rafd090_31_Caucasian_female_disgusted_frontal } \\
\text { Rafd090_56_Caucasian_female_disgusted_frontal } \\
\text { Rafd090_30_Caucasian_male_disgusted_frontal } \\
\text { Rafd090_24_Caucasian_male_disgusted_frontal }\end{array}$ \\
\hline sad & $\begin{array}{l}\text { Rafd090_01_Caucasian_female_sad_frontal } \\
\text { Rafd090_37_Caucasian_female_sad_frontal } \\
\text { Rafd090_03_Caucasian_male_sad_fronta } \\
\text { Rafd090_36_Caucasian_male_sad_frontal }\end{array}$ \\
\hline surprise & $\begin{array}{l}\text { Rafd090_58_Caucasian_female_surprised_frontal } \\
\text { Rafd090_57_Caucasian_female_surprised_frontal } \\
\text { Rafd090_46_Caucasian_male_surprised_frontal } \\
\text { Rafd090_05_Caucasian_male_surprised_frontal }\end{array}$ \\
\hline fear & $\begin{array}{l}\text { Rafd090_18_Caucasian_female_fearful_frontal } \\
\text { Rafd090_12_Caucasian_female_fearful_frontal } \\
\text { Rafd090_71_Caucasian_male_fearful_frontal } \\
\text { Rafd090_38_Caucasian_male_fearful_frontal }\end{array}$ \\
\hline
\end{tabular}

\begin{tabular}{|l|l|}
\hline angry & Rafd090_08_Caucasian_female_angry_frontal \\
& Rafd090_14_Caucasian_female_angry_frontal \\
& Rafd090_09_Caucasian_male_angry_frontal \\
& Rafd090_15_Caucasian_male_angry_frontal \\
\hline happy & Rafd090_22_Caucasian_female_happy_frontal \\
& Rafd090_61_Caucasian_female_happy_frontal \\
& Rafd090_07_Caucasian_male_happy_frontal \\
& Rafd090_21_Caucasian_male_happy_frontal
\end{tabular}

\section{Data Analysis}

Statistical analyses were conducted with the statistical package for the social sciences (SPSS). A mixed ANOVA was performed on the collected data to assess the label decoding accuracy and intensity ratings, with group (deaf and hearing subjects) as a between subjects variable, and emotional labels and intensity rating values as within subjects variables.
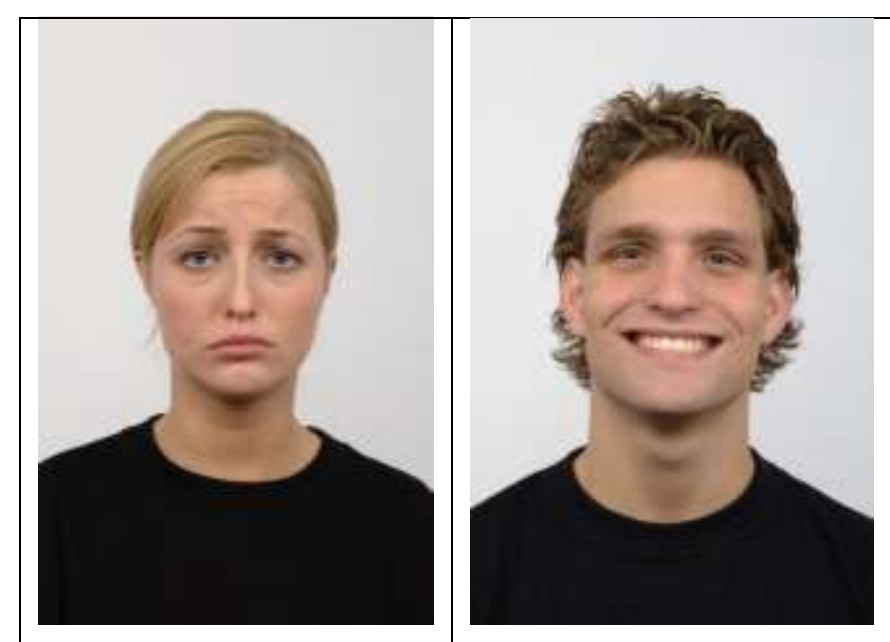

Fig. 1. An example of Dutch female and male face expressing sadness and happiness respectively.

\section{RESULTS}

\section{A. Decoding Accuracy}

Table II reports confusion matrices associated to the decoding accuracy performance of deaf and hearing subjects. Percentages are rounded to the nearest integer. On the principal diagonal it is possible to read the correct decoding accuracy, while on the rows it is possible, for each emotion, to assess the confusion made with the other assigned labels. The ANOVA analysis showed that there were significant differences in the performance of the deaf and hearing subjects $(F(1,26)=10.63$; $\mathrm{p}=.003)$. A significant interaction between Groups and Emotions $(\mathrm{F}(5,130)=3.67 ; \mathrm{p}=.004)$ was found. Bonferroni post hoc tests showed that the interaction was caused by the emotions of anger $(\mathrm{p}=.028)$, surprise $(\mathrm{p}=.003)$, and disgust 
$(\mathrm{p}<.001)$. For these three emotions the decoding accuracy of the deaf participants was significantly poorer than the hearing ones. Fig. 2 illustrates these differences, reporting the histogram of the decoding accuracy for deaf (white bars) and hearing (shaded bars) subjects, respectively.

It must be noticed that the only emotion that received a high degree of correct response for both deaf and hearing subjects was happiness. For the remaining emotions, only surprise $(76.8 \%)$ and disgust $(66.1 \%)$ obtained a percentage of correct decoding accuracy above chance in the group of hearing subjects. These data suggested that both deaf and hearing subjects performed poorly, even though the hearing group performed significantly better than the deaf one for disgust, surprise and anger. In order to explain these results, it can be interesting to report the percentage of correct accuracy obtained by Dutch hearing subjects with respect to the Italian ones. This comparison is possible since the Langner's et al. paper [27] provides additional documentation reporting, for each stimulus, the percentage of agreement among Dutch subjects participating to Langner's experiments. Unfortunately, it was not possible to perform a detailed statistical analysis comparing Italian and Dutch performance on the individual subject's responses, since the available additional documentation reports only the percentage of agreement obtained by all subjects. Therefore, the data that follow must be considered as exemplars and more data are needed to sustain the proposed argumentation.

\section{TABLE II}

Confusion matrices reporting on the columns the label accuracy for deaf and hearing subjects respectively.

\begin{tabular}{|l|c|c|c|c|c|c|c|c|}
\hline $\begin{array}{l}\text { Deaf } \\
\text { Label } \\
\begin{array}{l}\text { Acc. } \\
(\%)\end{array}\end{array}$ & sad & happy & fear & disgust & surprise & anger & other & $\begin{array}{l}\text { no } \\
\text { emo }\end{array}$ \\
\hline sad & $\mathbf{5 0}$ & 2 & 5 & 7 & 2 & 7 & 16 & 11 \\
\hline happy & & $\mathbf{8 6}$ & & & 4 & & 11 & \\
\hline fear & 4 & & $\mathbf{4 5}$ & 9 & 11 & 9 & 18 & 5 \\
\hline disgust & 4 & & 4 & $\mathbf{1 6}$ & 2 & 55 & 16 & 4 \\
\hline surprise & 2 & & 14 & 11 & $\mathbf{5 0}$ & 4 & 11 & 9 \\
\hline anger & 29 & & 5 & 11 & & $\mathbf{2 9}$ & 13 & 14 \\
\hline $\begin{array}{l}\text { Hearing } \\
\text { Label } \\
\text { Acc. } \\
(\mathbf{\%})\end{array}$ & sad & happy & fear & disgust & surprise & anger & other & $\begin{array}{l}\text { no } \\
\text { emo }\end{array}$ \\
\hline sad & $\mathbf{5 4}$ & 2 & 4 & 14 & 4 & 4 & 14 & 5 \\
\hline happy & & $\mathbf{9 1}$ & & & 2 & & 4 & 4 \\
\hline fear & 2 & & $\mathbf{5 0}$ & 14 & 20 & 2 & 13 & \\
\hline disgust & & & 2 & $\mathbf{6 6}$ & 5 & 7 & 7 & 13 \\
\hline surprise & & & 7 & 4 & $\mathbf{7 7}$ & 2 & 7 & 4 \\
\hline anger & 5 & & & 9 & 4 & $\mathbf{5 4}$ & 21 & 7 \\
\hline
\end{tabular}

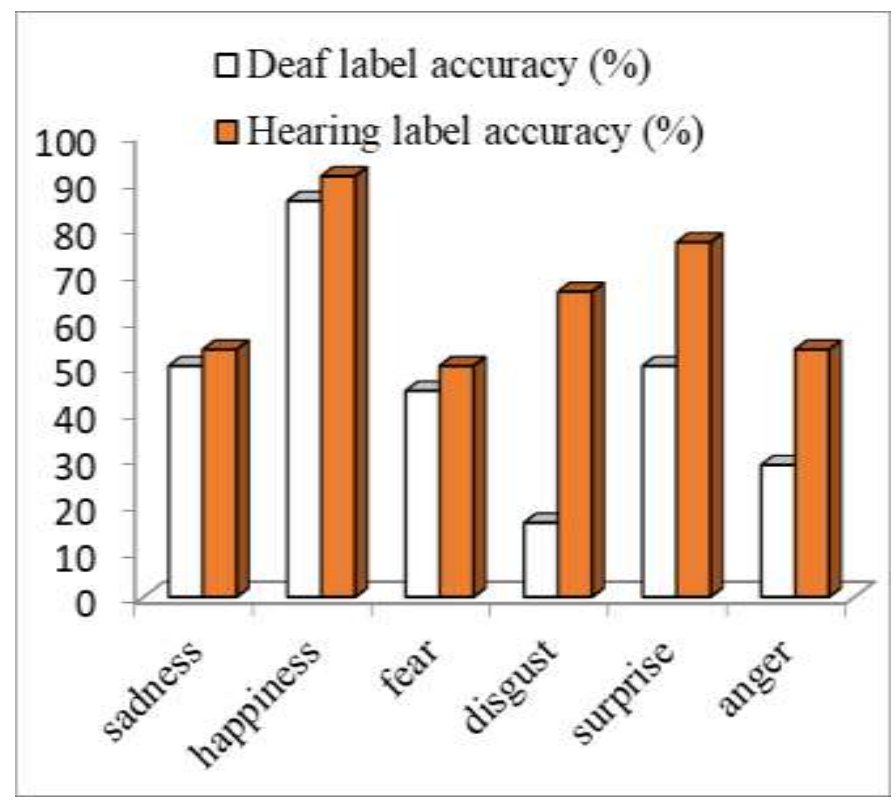

Fig. 2. Histogram of the decoding accuracy for deaf (white bars) and hearing (shaded bars) subjects. On the y-axis is reported the percentage of correct decoding accuracy.

Considering the data collected by [27] for Dutch and those collected by us for Italian subjects, a one tailed t-student test for two independent means was performed on the percentages of label agreements obtained on the same stimuli by Dutch and Italian participants. The results show that Italian mean label accuracy values are significantly lower than those obtained by Dutch ( $\mathrm{t}=3.15025, \mathrm{p}=.005)$. In particular, the decoding accuracy of Italian subjects is particularly poorer for sadness (54\% vs $91 \%$ of correct decoding accuracy for Italian and Dutch respectively), fear (50\% for Italian vs $86 \%$ of correct accuracy for Dutch), and anger (54\% for Italian vs $95 \%$ for Dutch). Fig. 3 illustrates these differences, reporting the histogram of the decoding accuracy for hearing Italian (white bars) and Dutch (shaded bars) subjects, respectively.

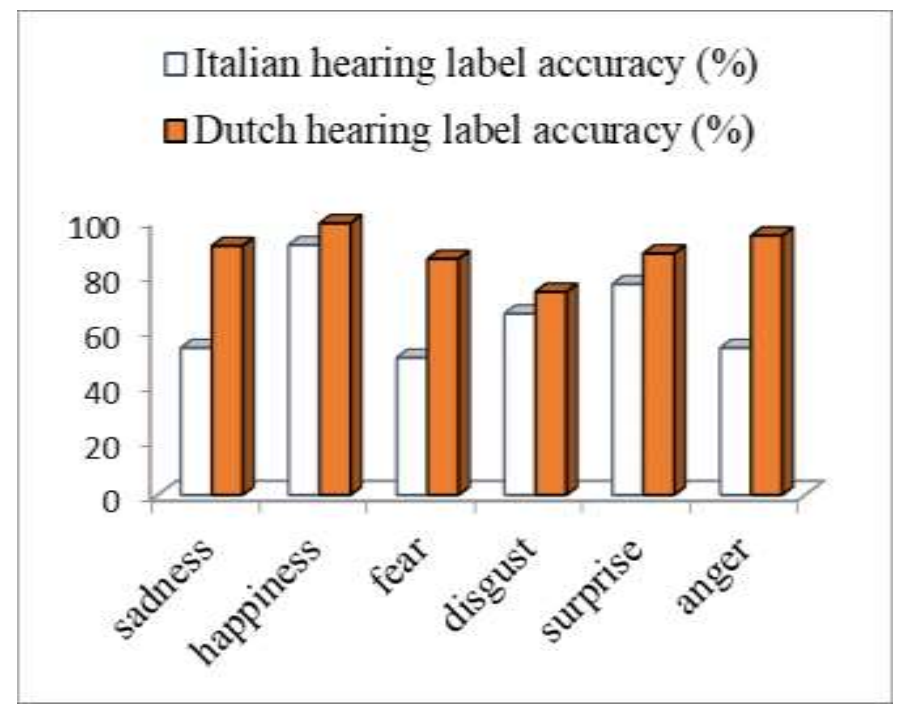


Fig. 3. Histogram of the percentage of decoding accuracy obtained by hearing Italian (white bars) and Dutch (shaded bars) subjects on the stimuli listed in Table I.

We suggest that these significant differences can be attributed to the fact that Dutch faces are expressively very different from those Italian subjects are familiar with. There are physical facial traits that do not conform to facial templates Italians learned in their social environment. Therefore, apart from stereotype expressions associated with smiles (as for happiness), or large eye openings (as for surprise) it is difficult for Italians to see in these not usually seen faces, emotions like fear, anger, and sadness. It can be argued that they need more closed eyes for anger, less lowered eyebrows for sadness, and more closed mouths for fear. However, with the data at the hand these are only speculations. More investigations are required. Even though it may appear "that Italian and Dutch culture are in the same culture region, with a lot of mutual influence" (according to a reviewer's comment), South Italian facial traits are strongly different from Dutch ones, and facial traits are part of what an individual learn about the sociocultural environment. Unfortunately, these aspects have been neglected in the quest for a universally shared facial emotional code. Nevertheless, these observations may explain why the accuracies in Table II are very low, even for hearing participants.

It would be interesting to investigate whether, other than for South Italian hearing subjects, similar results are observed within other "socio-cultural environments" that share with Dutch and Italian the label of "western culture", to compare with studies that examine very distinct cultures (e.g. [13]). To our knowledge, there are no studies reporting on similar investigations. There are however studies showing that facial somatic traits can influence the decoding of facial emotional expressions [2], [3], [26].

\section{B. Intensity Mean Ratings}

Table III reports confusion matrices associated with the mean rating intensity values for each categorical emotional face.

TABLE III

\begin{tabular}{|c|c|c|c|c|c|c|c|c|}
\hline \multicolumn{9}{|c|}{$\begin{array}{l}\text { Normalized confusion matrices reporting on columns the percentage of mean } \\
\text { intensity rating values obtained on the selected stimuli by deaf and hearing } \\
\text { subjects respectively. In order to show the amount of confusion with the other } \\
\text { emotions, percentages are computed, for each label, as the ratio of the sum of } \\
\text { the intensity ratings attributed to the selected label in each emotion category } \\
\text { (either the correct or incorrect one) over the sum of the intensity ratings } \\
\text { attributed to the correct and incorrect emotion category. }\end{array}$} \\
\hline $\begin{array}{l}\text { Deaf } \\
\text { Int. } \\
\text { scores } \\
(\%) \\
\end{array}$ & sad & happy & fear & disgust & surprise & anger & other & $\begin{array}{l}\text { no } \\
\text { emo }\end{array}$ \\
\hline sad & 55 & 3 & 5 & 10 & 2 & 5 & 8 & 12 \\
\hline happy & & 85 & & & 4 & & 11 & \\
\hline fear & 5 & & 52 & 8 & 11 & 8 & 11 & 5 \\
\hline disgust & 3 & & 3 & 20 & 1 & 56 & 17 & \\
\hline surprise & 2 & & 13 & 11 & 54 & 2 & 9 & 9 \\
\hline anger & 32 & & 6 & 10 & & 30 & 10 & 13 \\
\hline $\begin{array}{l}\text { Hearing } \\
\text { int. } \\
\text { scores } \\
(\%)\end{array}$ & sad & happy & fear & disgust & surprise & anger & other & $\begin{array}{l}\text { no } \\
\text { emo }\end{array}$ \\
\hline
\end{tabular}

\begin{tabular}{|l|l|l|l|l|l|l|l|l|}
\hline sad & $\mathbf{5 7}$ & 2 & 3 & 12 & 5 & 5 & 13 & 2 \\
\hline happy & & $\mathbf{9 2}$ & & & 2 & & 2 & 5 \\
\hline fear & & & $\mathbf{5 3}$ & 15 & 21 & 2 & 10 & \\
\hline disgust & & & 3 & $\mathbf{7 7}$ & 5 & 5 & 5 & 5 \\
\hline surprise & & & 9 & 2 & $\mathbf{7 6}$ & 3 & 7 & 3 \\
\hline anger & 6 & & & 8 & 4 & $\mathbf{5 9}$ & 17 & 6 \\
\hline
\end{tabular}

In order to display results as confusion matrices, these mean intensity values have been calculated as the ratio of the total intensity values attributed to each emotion category over the sum of the total intensity values attributed to the correct and mistaken emotion categories. What we have done is essentially a normalization of the intensity values. This normalization has been made only for display purposes. The effect on the data is to weaken statistically significant differences. However, the reported statistics have been made on the coarse intensity rating values attributed by participants to each emotion category. As for Table II, on the principal diagonal it is possible to read the mean intensity rating values assigned to each emotion, and on the rows the confusion made with the other emotional labels.

A mixed ANOVA analysis was performed on the intensity rating values, with group (deaf vs hearing subjects) as a between subjects variable, and facial emotional intensity rating values as within subjects variable. The ANOVA analysis shows significant differences between deaf and hearing subjects in assigning intensity values to the portrayed emotional faces $(F(1,26)=7.408 ; \mathrm{p}=.01)$. A significant interaction was also found between Group and Emotional Intensity $(\mathrm{F}(5,130)=4.479 ; \mathrm{p}=.001)$. Bonferroni post hoc tests show that deaf and hearing subjects differ significantly in rating intensity values of sadness $(\mathrm{p}=.016)$, fear $(\mathrm{p}=.007)$, surprise $(\mathrm{p}=.006)$, anger $(\mathrm{p}=.004)$, and disgust $(\mathrm{p}<.035)$. Fig. 4 illustrates these differences, reporting the histogram of the rough mean intensity rating values for deaf (white bars) and hearing (black bars) subjects respectively.

\section{$\square$ Deaf intensity rating values}

\section{- Hearing intensity rating values}

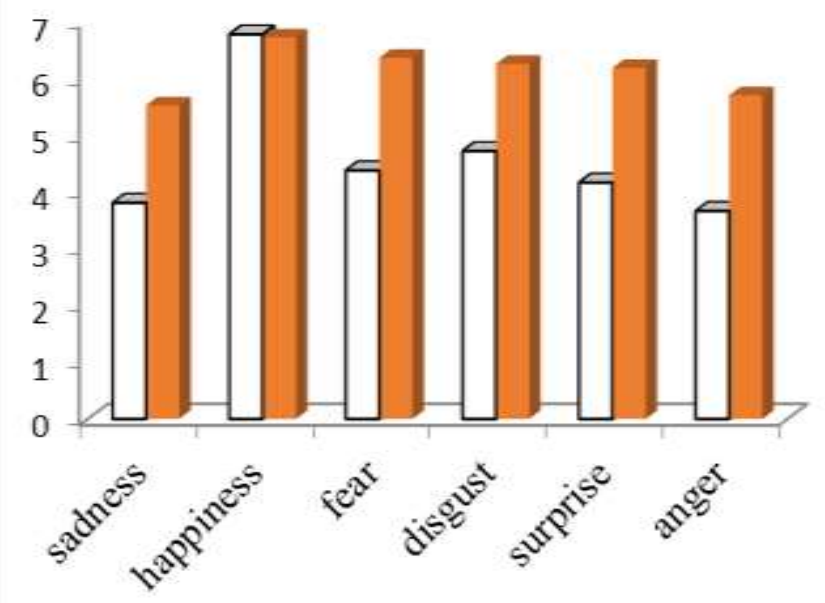

Fig. 4. Mean intensity rating values for deaf (white bars) and hearing (black bars) subjects. 
The general information emerging from these data is that deaf subjects perceive the selected faces less intensely emotionally portrayed than hearing subjects. This may suggests a reduced sensitivity of deaf with respect hearing subjects to the intensity dimension of emotions, due to the fact that sign languages exploit faces as communicative signs. In this context, the reduced sensitivity may be due to the fact that signers use faces semantically in order to communicate, for example, on something either disgusting, or fearful, or sad, without feeling fearful, sad, or disgusted. To this extent, the language attitude can influence the perception of emotional dimensions, as it has been shown for stereotype associations, prejudices, and gender differences [8], [21], [24], [25]. Since our language shapes our thoughts and behaviors [29], language's influence on the perception and decoding of emotional states merits extensive investigations. A comparison between hearing Italian and Dutch intensity rating values is not possible since a different Likert scale (from 0 to 9 vs one from 0 to 5 for Dutch) was used in this experiment.

\section{DisCUSSION AND CONCLUSION}

There are significant differences between deaf and hearing subjects in correctly decoding foreign facial emotional expressions of anger, disgust, and surprise. In addition, deaf showed significant differences in attributing a comparable degree of arousal (emotional intensity comparable to the hearing subjects) to facial expressions of disgust, surprise, fear, anger, and sadness. Deaf perform poorer than hearing subjects. Why is that?

Language specificity affects the ability to decode emotional faces. It may well be that in the case of deaf individuals using sign languages, the fact that facial expression contributes to the composition of semantic, and iconic dimension of the sign. It is possible that sign language use of facial expressions interferes with the interpretation of the same facial movements in their "raw" form as bearing emotion-related meaning [6], [13], [30].

It must be noticed that both deaf and hearing subjects perform poorly on five out of the six primary facial expressions of emotions (see Tables II and III). This suggests that typical face traits (Dutch faces) also affect the ability to correctly decode primary emotional facial expressions. If there are some universal templates for primary emotions, they are only related to happiness. The remaining facial expressions of primary emotions seem to be strongly affected by the emotional learned experience suggesting that South Italian emotional faces strongly diversify from Dutch emotional faces. Therefore, "even there exists a universal substrate tying the expression and decoding of emotional faces, this process can be less influential than it was previously hypothesized in regulating social emotional exchanges" ([4], p. 254).
These data bears evidence that in the quest for implementing future socially and emotional believable human-machine interactions, personal traits, cultural specificities, and contextual instances must be accounted for [10], [18], [19]. The envisaged promising applications in this field, such as those for the early interventions and management of cognitive and physical diseases, as well as, responsive and symbiotic personal assistants simulating human perception, attention, imagination, and emotions, need a holistic perspective. This perspective must take in hand how complex ICT interfaces must behave and appear in order to be social, and trustable, and which are, in a specific culture and language, the individual's social rules and cognitive competencies in terms of social shared meanings.

Future Cognitive Info Communication devices must undergo through an efficient modeling of user's communicative signals, competencies, beliefs and environmental information in order to provide relevant feedback and/or services [1]. On this line of effort, the topics offered by the CogInfocomm conference are extremely multidisciplinary covering matters such as faces and gesture recognition, cognitive networks, artificial cognitive capabilities of ITC systems, cognitive devices for rehabilitation and learning, intelligent vehicle transportations, augmented and virtual reality. In this multidisciplinary forum, scientists from different disciplines can meet and share results and ideas.

\section{ACKNOWLEDGMENT}

The authors thank the Ente Nazionale Sordi of Caserta, Italy for volunteering the participation of their associates to the experiment.

\section{REFERENCES}

[1] P. Baranyi, A. Csapo, and G. Sallai, Cognitive Infocommunications (CogInfoCom) Springer International Publishing Switzerland 2015. http://link.springer.com/book/10.1007\%2F978-3-319-19608-4

[2] D. V. Becker, D. T. Kenrick, S. L. Neuberg, K. C. Blackwell, and D. M. Smith, D.M. "The confounded nature of angry men and happy women," Journal of Personality and Social Psychology, vol. 92, pp. 179-190, 2007

[3] G. Bijlstra, R. W. Holland, and D. H. J. Wigboldus, "The social face of emotion recognition: Evaluations versus stereotypes," Journal of Experimental Social Psychology vol. 46, pp. 657-663, 2010.

[4] V. Capuano, M. T. Riviello, G. Cordasco, J. Mekyska, M. FaundezZanuy, A. Esposito, "Assessing natural emotional facial expressions: An evaluation of the I.Vi.T.E. database". In von Dieter Mehnert H, Kordon U, Wolff M (Eds) Rüdiger Hoffmann zum 65. Geburtstag Systemtheorie Signalverarbeitung Sprachtechnologie, TUDpress 2013. Kartoniert, pp. 249-255

[5] O. Crasborn, E. vanderKooij, D. Waters, B. Woll, and J. Mesch "Frequency distribution and spreading behavior of different types of mouth actions in three sign languages," SignLang.Linguist, vol. 11, pp. 45-67, 2008.

[6] C. Cecchetto, C. Geraci, and S. Zucchi, "Strategies of relativization in Italian Sign Language". Natural Language and Linguistic Theory vol. 24 , pp. $945-975,2006$ 
[7] S. Dachkovsky, and W. Sandler, "Visual intonation in the prosody of a Sign Language.” Lang.Speech, vol. 52, pp. 287-314, 2009.

[8] R. Dotsch, H. J. Wigboldus, O. Langner, and A. van Knippenberg, "Ethnic outgroup faces are biased in the prejudiced mind," Psychological Science, vol. 19, pp. 978-980, 2008.

[9] M. J. Dyck, C. Farrugia, I. M. Shochet, and M. Holmes-Brown, "Emotion recognition understanding ability in hearing or vision impaired children: Do sounds, sights or words make the difference?," Journal of Child Psychology and Psychiatry, vol. 45, pp. 789-800, 2004.

[10] H. A. Elfenbein, and N. Ambady, "On the universality and cultural specificity of emotion recognition: A meta-analysis," Psychological Bulletin, vol. 128, pp.205-235, 2002.

[11] P. Ekman, "An argument for basic emotions," Cognition and Emotion, vol. 6, pp.169-200, 1992

[12] P. Ekman, and W. V. Friesen, "Constants across cultures in the face and emotion," Journal of Personality and Social Psychology vol. 17(2), pp. 124-129, 1971.

[13] E. A. Elliott, and A. M. Jacobs, "Facial expressions, emotions, and sign languages". Frontiers in Psychology, 11 March 2013 https://doi.org/10.3389/fpsyg.2013.00115

[14] A. Esposito, "The perceptual and cognitive role of visual and auditory channels in conveying emotional information," Cognitive Computation, vol. 1, pp. 268-278, 2009.

[15] A. Esposito, D. Palumbo, and A. Troncone, "The influence of the attachment style on the decoding accuracy of emotional vocal expressions," Cognitive Computation, vol. 6(4), pp. 699-707, 2014.

[16] A. Esposito, D. Palumbo, and A. Troncone, "Effects of narrative identities and attachment style on the individual's ability to categorize emotional voices. In Advances in Neural Networks: Computational and Theoretical Issues, S. Bassis, A. Esposito, and F.C. Morabito Eds, Springer International Publishing. SIST series, vol. 37, 2015, pp. 265 272.

[17] A. Esposito, A. M. Esposito, and C. Vogel, "Needs and challenges in human computer interaction for processing social emotional information," Pattern Recognition Letters, vol. 66, pp. 41-51, 2015.

[18] A. Esposito, and L. C. Jain, "Modeling social signals and contexts in robotic socially believable behaving systems,". In Toward Robotic Socially Believable Behaving Systems, vol. II, Intelligent Systems Reference Library, ISRL 106, A. Esposito and L. C. Jain Eds., Springer International Publishing Switzerland, 2016, pp. 5-13.

[19] A. Esposito, and L. C. Jain, "Modeling emotions in robotic socially believable behaving systems. In Toward Robotic Socially Believable Behaving Systems, vol. I, Intelligent Systems Reference Library, ISRL 105, A. Esposito and L.C. Jain Eds., Springer International Publishing Switzerland, 2016, pp. 9-15.

[20] A. Esposito, and A. Troncone, "Differences between alcoholic and non alcoholic individuals in the recognition of vocal emotional stimuli: A case-control study," In Proceeding of the $7^{\text {th }}$ IEEE international Conference on Cognitive InfoCommunications, Wroclaw, 16-18 Oct 2016, pp.19-23
[21] A. H. Fischer, P. M. Rodriquez Mosquera, A. E. M. Vianen, and A. S. R. Manstead, "Gender and culture differences in emotion," Emotion, vol, 4, pp. 87-94, 2004.

[22] C. D. Gray, J. A. Hosie, P. A. Russell, and E. A. Ormel, "Emotiona development in deaf children: Facial expressions, display rules, and theory of mind," In Context, cognition and deafness, M. D. Clark, M. Marschark, and M. Karchmer Eds., Washington, DC: Gallaudet University Press, 2001, pp. 135-157.

[23] J. A. Hosie, C. D. Gray, C. D. Russell, C. Scott, C., and N. Hunter, "The matching of facial expressions by deaf and hearing children and their production and comprehension of emotion labels," Motivation and Emotion, vol, 22, pp. 293-313, 1998.

[24] K. Hugenberg, and S. Sczesny, "On wonderful women and seeing smiles: Social categorization moderates the happy face response latency advantage," Social Cognition, vol. 24, pp. 516-539, 2006.

[25] K. Hugenberg, and G. V. Bodenhausen, "Facing prejudice: Implicit prejudice and the perception of facial threat," Psychological Science, vol. 14, pp. 640-643, 2003.

[26] K. Hugenberg, "Social categorization and the perception of facial threat: Target race moderates the response latency advantage for happy faces," Emotion, vol. 5, pp. 267-276, 2005 .

[27] O. Langner, R. Dotsch, G. Bijlstra, D. H. J. Wigboldus, S. T. Hawk, and A. D. van Knippenberg, "Presentation and validation of the Radboud Faces Database," Cognition \& Emotion, vol. 24(8), pp.1377-1388, 2010

[28] N. M. Maldonato, and S. Dell'Orco, "Making decision under uncertainty, emotions, risk and biases" In Advances in Neural Networks: Computational and Theoretical Issues, S. Bassis, A. Esposito, and F. C. Morabito Eds., Springer International Publishing Switzerland, SIST series, vol. 37, 2015, pp. 293-302.

[29] O. Y. U. Mykhailyuk, and H. Y. A. Pohlod, "The languages we speak affect our perceptions of the world," Journal of Vasyl Stefanyk Precarpathian National University, http://jpnu.pu.if.ua, vol. 2 (2-3), pp. 36-41, 2015.

[30] J. S. Reilly, M. Mcintire, and U. Bellugi, "The acquisition of conditionals in American Sign Language: Grammaticized facial expressions," Applied Psycholinguistics, vol. 11(4), pp.369-392, 1990.

[31] M. T. Riviello, and A. Esposito, "On the perception of dynamic emotional expressions: A cross-cultural comparison," In SpringerBriefs in Cognitive Computation, A. Hussain Ed., Springer International Publishing, vol. 6, pp. 1-54, 2016.

[32] W. Sandler, "Symbiotic symbolization by hand and mouth in sign language," Semiotica, vol. 174, pp. 241-275, 2009.

[33] R. B. Wilbur, "Effects of varying rate of signing on ASL manual signs and non-manual markers," Language and Speech, vol. 52, pp. 245-285, 2009.

[34] B. Woll, "The sign that dares to speak its name: Echophonology in British Sign Language (BSL)," In The Hands are the Head of the Mouth, P. Boyes Braem, and R. Sutton-Spence Eds., Hamburg:Signum-Verlag 2009,pp.87-98. 